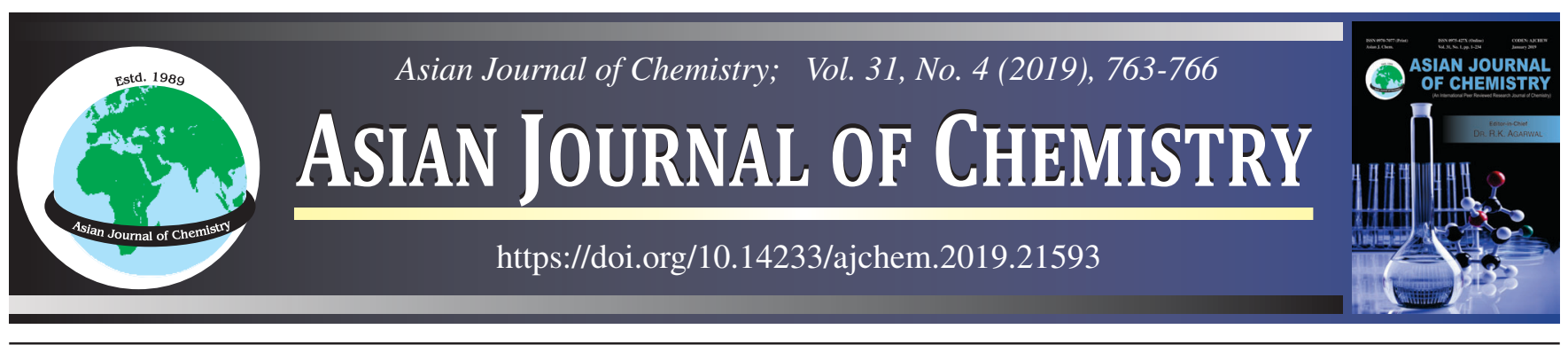

\title{
Determination of Glucose Concentration in Anaerobic Acidification Cultures by Portable Glucose Monitoring System
}

\section{DARWIN}

Department of Agricultural Engineering, Syiah Kuala University, Banda Aceh 23111, Indonesia

Corresponding author: E-mail: darwin_ae@unsyiah.ac.id

Received: 18 July 2018;

Accepted: 18 November 2018;

Published online: 27 February 2019;

AJC-19282

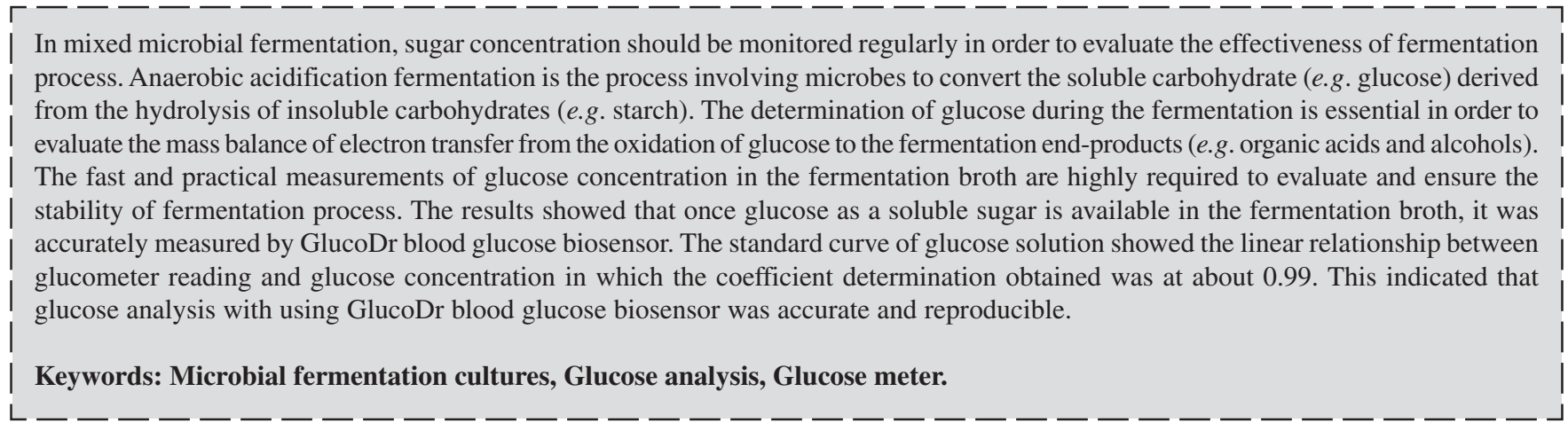

\section{INTRODUCTION}

The development of practical and accurate methods for monitoring glucose levels in microbial fermentation cultures has significant contribution for improving fermentation process and analysis protocols for both researchers and industrial fermentation engineers. The use of portable glucose meter in industrial fermentation processes would significantly assist the operators to monitor and determine glucose concentration in fermentation broth regularly [1]. This is important since it could potentially minimize the problems that may appear during the fermentation process, such as low yield and low productivity of the end-product formation. Hence, those problems could be prevented in advance by controlling and monitoring glucose concentration during the course of fermentation.

Monitoring soluble sugar during the carbohydrate fermentation is essential since high concentration and/or accumulation of soluble sugar in the fermentation broth could also affect the growth of bacteria [2,3]. Uncontrolled soluble sugar accumulation in the fermentation broth, could also lead to the decrease of productivity and yields of fermentation end-products [4]. This condition usually occurs in rumen fermenting carbohydrates $[5,6]$. Therefore, a practical and reliable method for monitoring glucose concentration in the fermentation broths is highly required. Some methods for the determination of glucose concentration in fermentation broth has been developed by using some methods such as colorimetric [7] and HPLC [8]. Those two methods could be considered as non-practical methods due to time consuming and required some additional pre-treatments and preparations such as heating and chemical pre-treatments (e.g. use of anthrone reagent, ethanol and sulfuric acid) $[9,10]$.

Glucose meter initially was used to measure blood glucose for getting quick as well as rapid results. Thus, blood glucose determination using glucose meter could help people with diabetes for avoiding acute and late complications of their illness [11]. Study conducted by Hönes et al. [12] mentioned that test strips are the main component that determined accurate measurement of glucose concentration on blood samples [12]. Their effectiveness on determining glucose concentration is due to

This is an open access journal, and articles are distributed under the terms of the Creative Commons Attribution-NonCommercial-ShareAlike 4.0 (CC BY-NC-SA 4.0) International License which allows readers to freely read, download, copy, distribute, print, search, or link to the full texts of its articles and to use them for any other lawful non-commercial purpose as long as the original source is duly acknowledged. 
the fact that the strips consist of enzymes, coenzymes, mediators and indicators in the form of a dry layer. Those essential components could be able to convert glucose concentration into a signal, which could be readable by the glucose meter. The accuracy, precision, specificity and measurement speed in determination of glucose concentration would highly be influenced by test strip chemistry and design [12].

As the measurement of glucose concentration in the blood sample using glucose meter are considered as an accurate method, it could potentially be developed further and applied in other biological samples. A study conducted by Flavigny [13] showed that glucose meter could accurately determine glucose concentration on microbial cultures, and the glucose meter used in the study was AccuCheck Active (Roche). The study measured glucose concentration from glycogen stored in microbial cultures of wastewater. The glycogen had been firstly hydrolyzed to glucose prior to the determination of its concentration.

The present study aimed to evaluate the effectiveness of glucose meter (GlucoDr) in the determination of glucose concentration on fermentation samples particularly in the samples of anaerobic acidification samples. This is highly useful as the analysis of glucose concentration on microbial fermentation samples using glucose meter with strips would provide rapid, accurate and reliable results. Therefore, the study on this development method may have a widely significant impact on improving research and development in industrial fermentation processes.

\section{EXPERIMENTAL}

A GlucoDr ${ }^{\mathrm{TM}}$ (allmedicus) blood glucose monitoring system with an assay method of electrochemical method (Gold electrode) was utilized. The model of glucose meter used was BioSensor AGM-2100. The sample volume allowed was $4 \mu \mathrm{L}$ (minimum $2.5 \mu \mathrm{L}$ ) with the measurement time of $11 \mathrm{~s}$, and the calibration applied was plasma-equivalent. As portable equipment, the glucose meter used battery two 3-volt lithium batteries (coin cell type CR2032). The use of glucose meter was in corresponding test strips. The strip used in this glucometer was Gold Plated Test Strip (allmedicus).

Standard solution: Standard stock solution of $50 \mathrm{mM}$ glucose was prepared from the lab-grade glucose, D-(+)-glucose anhydrous (VWR BDH Prolabo Chemicals). From this stock solution, desired standard solution or various ranges of glucose concentrations were prepared. Standard curves for glucose were plotted from the glucose meter reading responses to the standard solution, conducted as mentioned previous, with concentrations ranging from 0 to $30 \mathrm{mM}$.

Analysis: Samples were taken from anaerobic acidification process of Saccharomyces cerevisiae and Lactobacillus acidophilus cultures fed with the sugarcane as substrate. Samples of $2 \mathrm{~mL}$ fermentation broth were drawn from the anaerobic acidification reactors from the time of 0 to $24 \mathrm{~h}$ of incubation. As the measurement range of the glucose meter was from 20 to $600 \mathrm{mg} / \mathrm{dL}(1.1-33.3 \mathrm{mmol} / \mathrm{L})$ of glucose concentration, samples for glucose analysis were diluted 10 times prior to dropping it on the test strip. The determination of glucose concentration using glucometer was carried out under room temperature.

\section{RESULTS AND DISCUSSION}

To understand the conversion of soluble organic compounds particularly sugar within fermentation process, the monitoring of the main soluble sugar (glucose) is extremely important. In order to evaluate the effectiveness of glucose measurement method using glucose meter and its reproducibility in glucose analysis, different concentrations of prepared glucose standard solution were used for investigation. A set of glucose standard solutions $(0-30 \mathrm{mmol} / \mathrm{L})$ was prepared for determining the relationship between the glucose meter readings and the concentrations of glucose solution.

The results showed that the glucose meter readings of standard solution containing glucose generated linear standard curve (Fig. 1). The coefficient of determination $\left(\mathrm{R}^{2}\right)$ for the standard curve obtained from glucose analyzed with different ranges of concentration was in general at 0.99 . This suggests that the measurement of glucose concentration in the solution containing glucose was accurate and reproducible and could potentially be used for measuring glucose content in the fermentation samples.

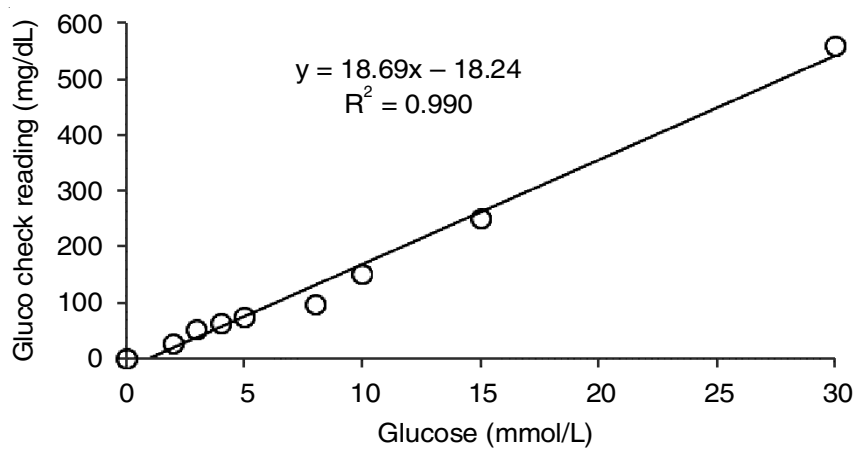

Fig. 1. Linearity of the glucose standard solution measured with the GlucoDr-Check

The above glucose measurement method was tested for relevant real world application using anaerobic acidification samples. Two types of samples were taken from the two different fermentation broths including samples of anaerobic acidification of sugarcane inoculated with both L. acidophilus and $S$. cerevisiae. The results showed that the glucose measurement on both fermentation samples could provide accurate glucose concentration during the fermentation of sugarcane in which the trends shown were a decrease of soluble sugar within $24 \mathrm{~h}$ of incubation (Figs. 2 and 3).

The results also revealed that the total conversions of glucose as soluble sugar within $24 \mathrm{~h}$ of incubation in both Lactobacillus acidophilus and Saccharomyces cerevisiae cultures were 48.3 and $78.45 \%$, respectively. Further, the soluble sugar conversion rate in both fermentation samples of $L$. acidophilus and $S$. cerevisiae cultures were 5.53 and $9.07 \mathrm{mmol} / \mathrm{L}$ per hour (Figs. 2 and 3). This indicated that determination of glucose concentration in the fermentation samples could effectively be carried out by using blood glucometer.

The glucose meter has been accepted as a practical and accurate method for determining the blood sugar [14-16]. Beecham et al. [17] showed that the glucose meter could be useful tools for analyzing glucose in the field or laboratory. 


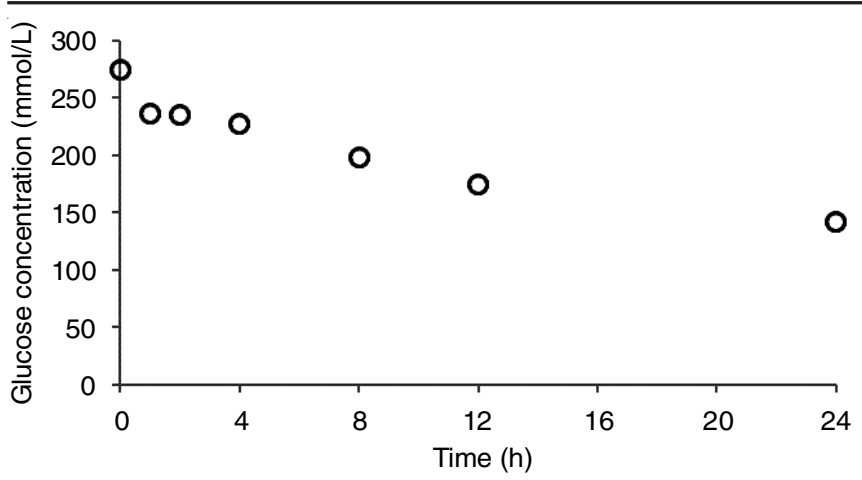

Fig. 2. Glucose analysis of sugarcane fermented with Lactobacillus acidophilus

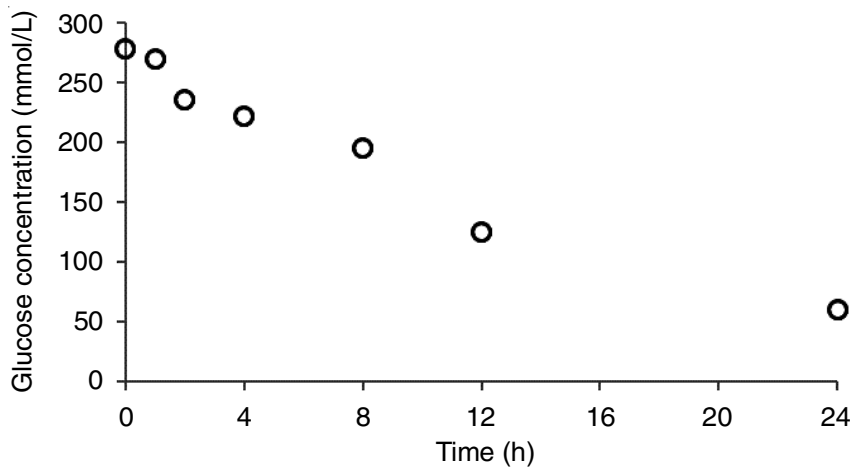

Fig. 3. Glucose analysis of sugarcane fermented with yeast

They used portable glucose meter for catfish research in which they compared the analysis of glucose using glucose meter and established laboratory method to evaluate fish metabolism comprehensively. The study revealed that the glucose meter showed low variability and little deviation among replicates. However, the data of glucose concentration obtained from glucose meter method are consistently lower than those are analyzed with an established laboratory method using spectrophotometer.

As the analysis of glucose concentration using glucose meter could provide rapid results, the use of this tool for measuring sugar concentration in other biological samples such as anaerobic digestion and/or acidification cultures [18,19] would potentially be applied, and it would be useful to reduce time for sample analysis. Flavigny [13] also revealed that glucose meter could be used for analyzing sugar content in microbial cultures of wastewater treatment, and generated reproducible results. The determination of glucose concentration in other biological samples such as fermentation samples using glucose meter has been scarcely conducted. Study conducted by Thornton and Brown [1] showed blood glucose biosensor had been successfully used to measure glucose concentrations in fermentation broths. Further, the study revealed that a highly sensitive linear calibration was obtained between the glucose concentration and the biosensor reading, which correlated well with a reducing sugar assay. In the study, they used Exactech glucose meter while the current study used the blood glucose meter to measure glucose concentration in the samples of anaerobic acidification of sugarcane.

The current study showed that the glucose analysis using glucometer with strips could also be used as an alternative and reliable method for determining glucose concentration in the samples of sugarcane fermentation (Figs. 2 and 3). The results of the present study showed that the glucose meter could effectively measure the actual glucose concentration in each sample of fermentation broth. It could be practically used for providing rapid results and quick overview in regards to the conversion process of glucose present in the sugarcane into metabolites. Therefore, the type of fermentation pathways during the time of fermentation process could thoroughly be evaluated.

The reliability of glucose readings was obtained as the analysis which has been proved with the standard curve showing the reproducibility of glucose analysis. In this study, the linear relationship was obtained between glucose concentration and glucose readings of glucose meter. Hence, a glucose meter could be used to monitor and follow the course of fermentation process, and could be applied to carry out mass balance analysis and/ or stoichiometric calculations [1].

\section{Conclusion}

Determination of glucose concentrations in the samples of anaerobic acidification of sugarcane could successfully be accomplished by using blood glucose meter. Prior to the sample analysis, a highly sensitive calibration was acquired between the glucose concentration and the glucose meter reading. The linear relationship was shown with high coefficient determination $\left(\mathrm{R}^{2}\right)$ of 0.99 .

\section{ACKNOWLEDGEMENTS}

The author acknowledges the financial support provided by Syiah Kuala University, Banda Aceh, Indonesia through $\mathrm{H}$-index research program coordinated by the Institute for Research and Community Services, UNSYIAH. The author also thank to Ulfa Triovanta and Ridho Rinaldi for helping in the collection of samples.

\section{CONFLICT OF INTEREST}

The author declares that there is no conflict of interests regarding the publication of this article.

\section{REFERENCES}

1. A.J. Thornton and D.E. Brown, Biotechnol. Tech., 5, 363 (1991); https://doi.org/10.1007/BF00185015.

2. J.B. Russell, C.J. Sniffen and P.J. Van Soest, J. Dairy Sci., 66, 763 (1983); https://doi.org/10.3168/jds.S0022-0302(83)81856-6.

3. B.A. Williams, M.W. Bosch, H. Boer, M.W. Verstegen and S. Tamminga, Anim. Feed Sci. Technol., 123-124, 445 (2005);

https://doi.org/10.1016/j.anifeedsci.2005.04.031.

4. J.B. Russell, J. Anim. Sci., 76, 1955 (1998); https://doi.org/10.2527/1998.7671955x.

5. J.B. Russell and J.L. Rychlik, Science, 292, 1119 (2001); https://doi.org/10.1126/science.1058830.

6. Darwin, A. Barnes and R. Cord-Ruwisch, Ann. Microbiol., 68, 1 (2018); https://doi.org/10.1007/s13213-017-1307-X.

7. Y.L. Dong, H.G. Zhang, Z.U. Rahman, L. Su, X.J. Chen, J. Hu and X.G. Chen, Nanoscale, 4, 3969 (2012); https://doi.org/10.1039/c2nr12109c.

8. Y. Liu, Y. Ying, H. Yu and X. Fu, J. Agric. Food Chem., 54, 2810 (2006); https://doi.org/10.1021/jf052889e.

9. A. Saifer and S. Gerstenfeld, Transl. Res., 51, 448 (1958).

10. T. Masuko, A. Minami, N. Iwasaki, T. Majima, S.I. Nishimura and Y.C. Lee, Anal. Biochem., 339, 69 (2005); https://doi.org/10.1016/j.ab.2004.12.001. 
11. K. Tonyushkina and J.H. Nichols, J. Diabetes Sci. Technol., 3, 971 (2009); https://doi.org/10.1177/193229680900300446.

12. J. Hönes, P. Müller and N. Surridge, Diabetes Technol. Ther, 10, 1 (2008); https://doi.org/10.1089/dia.2008.0005.

13. R. Flavigny, J. Microbiol. Methods, 100, 91 (2014); https://doi.org/10.1016/j.mimet.2014.03.002.

14. C.D. Critchell, V. Savarese, A. Callahan, C. Aboud, S. Jabbour and P. Marik, Intensive Care Med., 33, 2079 (2007); https://doi.org/10.1007/s00134-007-0835-4.

15. Z. Trajanoski, G.A. Brunner, R.J. Gfrerer, P. Wach and T.R. Pieber, Diabetes Care, 19, 1412 (1996); https://doi.org/10.2337/diacare.19.12.1412.
16. L.E. Thomas, M.P. Kane, G. Bakst, R.S. Busch, R.A. Hamilton and J.M. Abelseth, Diabetes Technol. Ther, 10, 102 (2008); https://doi.org/10.1089/dia.2007.0244.

17. R.V. Beecham, B.C. Small and C.D.N. Minchew, Am. J. Aquac., 68, 291 (2006); https://doi.org/10.1577/A05-074.1.

18. Darwin, J.J. Cheng, J. Gontupil and Z. Liu, Int. J. Environ. Waste Manag., 17, 71 (2016); https://doi.org/10.1504/IJEWM.2016.076432.

19. Darwin, W. Charles and R. Cord-Ruwisch, J. Chromatogr. Sci., 56, 1 (2018);

https://doi.org/10.1093/chromsci/bmx086. 